\title{
Template-Free Assembly in Living Bacterial Suspension under an External Electric Field
}

\author{
Kunal Samantaray, ${ }^{\dagger, \# ~ P r a c h i ~ B h o l, ~}{ }^{\ddagger}, \#$ Bhabani Sahoo, ${ }^{\S}$ Subrat Kumar Barik, ${ }^{\dagger}$ Kiran Jathavedan," \\ Bikash Ranjan Sahu, ${ }^{\dagger}$ Mrutyunjay Suar, ${ }^{\dagger}$ Suresh K. Bhat, ${ }^{\|}$and Priti Sundar Mohanty ${ }^{* \dagger, \dagger, \perp \odot}$ \\ ${ }^{\dagger}$ School of Biotechnology and ${ }^{\ddagger}$ School of Applied Sciences, KIIT University, Bhubaneswar 751024, India \\ ${ }^{\S}$ Institute of Life Science, Bhubaneswar 751023, India \\ "Polymer Science \& Engineering Division, CSIR-National Chemical Laboratory, Pune 411008, India
}

\section{Supporting Information}

\begin{abstract}
Although template-assisted self-assembly methods are very popular in materials and biological systems, they have certain limitations such as lack of tunability and switchable functionality because of the irreversible association of cells and their matrix components. With an aim to achieve more tunability, we have made an attempt to investigate the self-assembly behavior of rod-shaped living bacteria subjected to an external alternating electric field using confocal microscopy. We demonstrate that rod-shaped living bacteria dispersed in a low salinity aqueous medium form different types of reversible freely suspended structures when subjected to an external alternating electric field. At low field strength, an oriented phase is observed where individual bacterium orients with its major axis aligned along the field direction. At intermediate field strength, bacteria align in the form of one-dimensional (1D) chains that lie along the field direction. Further, at high field strength, more bacteria associate with these 1D chains laterally to form a two-dimensional (2D) array. At higher bacterial concentration, these field-induced 2D arrays extend to form three-dimensional columnar structures. These results are discussed in the context of previously reported studies on bacterial self-assembly.
\end{abstract}

\section{INTRODUCTION}

Studying self-assembly behavior in live bacterial cells of different shapes and sizes can lead to designing new biomaterials with interesting structural properties. So far, most of the studies on bacterial organizations are templatebased, where liquid crystals, ${ }^{1,2} \mathrm{DNA},{ }^{3,4}$ nanowires, ${ }^{5}$ or surfaces ${ }^{6}$ act as physical templates. Although template-based methods are also very popular in the area of nanoparticle assemblies ${ }^{7-9}$ and produce highly ordered permanent structures, they have certain limitations such as switchable functionality with rapid and reversible action.

On the other hand, in a template-free method, the selfassembly processes are studied in a bulk medium without the use of any physical template and the assembly processes can be controlled by parameters such as particle density, temperature, $\mathrm{pH}$, and electric and magnetic fields. External electric or magnetic field has advantages over other parameters with respect to fast switching on/off action. Moreover, the selfassembly can be tuned statically or dynamically. Although external fields have been used extensively to study phase transitions and related self-assembly in different types of colloidal or nanoparticle systems, ${ }^{10-17}$ their use in the selfassembly of biological cells is very rare. ${ }^{18-21}$ Velev group ${ }^{18}$ have studied field-induced coassembly of particles with yeast and fibroblast cells in the low frequency range (hertz to kilohertz), where they have found one-dimensional (1D) chains and two- dimensional (2D) arrays. Kang and Dhont ${ }^{19,20}$ have investigated electric-field-induced phase transitions of fd virus at low ionic strength and at low frequency (hertz to kilohertz range). These studies have observed various interesting phases such as nematic, striped, and a dynamical state. To our knowledge, no studies have been carried out yet on template-free assembly of micron-size living bacterial cells under an external alternating electric field (ac). Living bacterial cells can be considered to be complex dielectric particles ${ }^{22}$ containing different components such as vacuoles, nucleoid flagella, membrane, and so forth. Therefore, the dielectric contributions from these components and their intricate interplay with an external ac could generate novel field-induced structures. In addition, we know that many bacteria form biofilms while adapting to their host environment and experience isotropic and anisotropic forces that arise from the interacting environment itself. ${ }^{4,23}$ Hence, self-assembly of bacteria under an external electric field could be a good model to address how these anisotropic forces influence structural and dynamic properties of bacterial assembly.

Received: December 22, 2016

Accepted: March 6, 2017

Published: March 17, 2017 


\section{RESULTS AND DISCUSSION}

Zero-Field Characterizations of Bacterial Suspension. In the current study, we use green-fluorescence-protein-tagged rod-shaped Salmonella Typhimurium (Strain SB300) 24,25 bacterium with an average aspect ratio $(L / d)$ of $5 \pm 1$ ( $L$ is length $=2.5 \pm 0.9 \mu \mathrm{m}$ and $d$ is width $=0.5 \pm 0.3 \mu \mathrm{m})$. From dynamic light-scattering studies, the estimated average hydrodynamic radius and polydispersity are $1176 \mathrm{~nm}$ and 15\%, respectively (see Figure S2 for details). The details of bacterial culture preparation, its characterization, and the growth curve are provided in Supporting Information. All our field experiments are carried out by collecting samples from the stationary phase (at $16 \mathrm{~h}$ ) of growth culture (see Figure S1A). The bacterial samples were purified by washing several times with deionized Milli- $Q$ water by centrifugation at $3000 \mathrm{rpm}$. Ion-exchange resins were added to further deionize the bacterial suspension. Under deionized state, bacterial cells have a zeta potential of $-30 \mathrm{mV}$ (see Figure S1B). The field studies are carried out at room temperature $\left(T=20{ }^{\circ} \mathrm{C}\right)$. Importantly, we make sure that bacterial growth is restricted in deionized aqueous medium, and the field values are low enough to not damage or kill the cells. We implement two different schemes for our field experiments carried out using confocal microscope. Usually, the microscopy resolution along $z$ is poorer than the $x y$ resolution. Therefore, a fully three dimensional (3D) reconstruction of the structure and viewing it along the field direction are very challenging using Scheme 2. Hence, we use Scheme 1 to perform field experiments with the field along the $x$ direction.

Scheme 1. AC Field Is Applied in the Image Plane along the $x$-Axis as Shown in the Figure ${ }^{a}$

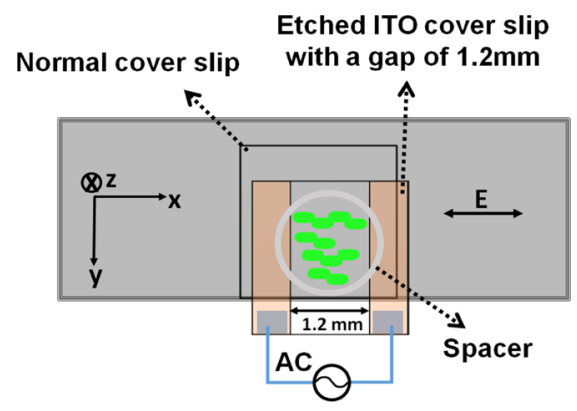

${ }^{a}$ Bacterial suspension is kept between two cover slips separated by a spacer of thickness 120 micron. One of the cover slips is coated with conductive indium tin oxide (ITO) layer and is etched with a gap of $1.2 \mathrm{~mm}$. Under an ac field, bacteria align along the field direction in the form of chains, and we see them in the image plane itself. The $z$ axis is perpendicular to the image $(x y)$ plane.

Electric Field Studies. All our field experiments are carried out at a fixed frequency of $2 \mathrm{MHz}$ by varying bacterial concentration and electric field strength. From our frequencydependence studies at constant electric field strength, we have verified that the field-induced structure formations are stronger above $1 \mathrm{MHz}$ (see Figure S3).

By lowering the frequency, the structures become weak and start melting at around $900-800 \mathrm{kHz}$. Upon further lowering the frequency, the elongated structures completely melt at around $150 \mathrm{kHz}$, and all bacteria with their long axis remain oriented along the field direction.
In a deionized suspension, bacteria have surface charges. It is possible that in the low frequency regime (in the kilohertz regime), the double-layer polarization dominates as the diffuse charge clouds around the bacterial cells follow the alternating cycle. Electric field-induced phase transitions due to doublelayer polarizations (in the kilohertz regime) can also be seen in other systems ${ }^{13,15,18,19}$ such as colloids, fd viruses, and cells. However, at a high frequency (in the megahertz range), the double-layer charges do not respond and only dielectric polarizations occur as mentioned in earlier electric field studies on colloidal systems. ${ }^{12,16}$ In the previous theoretical studies, biological cells are often modeled as dielectric particles covered with a membrane. ${ }^{26,27}$ The effective permittivity of the whole cell is due to the contributions from the permittivity of cytoplasm and membrane.

Therefore, it is likely that the dielectric polarization in the megahertz regime is a contribution of polarizations of the different components of bacterial cells including the membranes. However, to understand the details of the frequencydependent mechanisms of polarization in our bacterial suspension, we will need to carry out impedance spectroscopy: such studies are planned for the future. In the current study, we look at the electric field assembly only at a fixed frequency of 2 $\mathrm{MHz}$.

The field experiments performed as outlined in Scheme 1 are demonstrated in Figures 1 and 2 at a bacterial concentration of
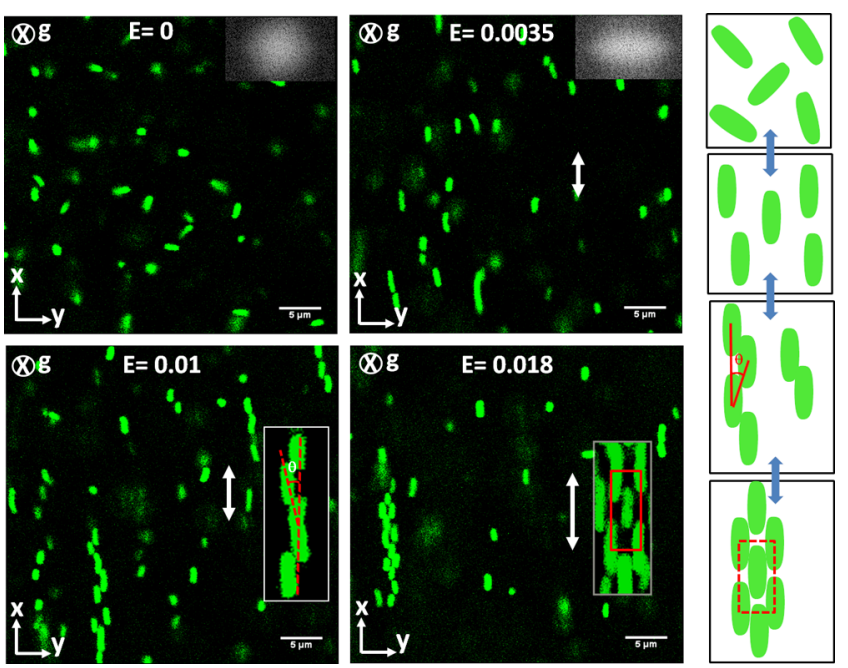

Figure 1. 2D confocal laser scanning microscopy (CLSM) images of bacterial suspension (at a concentration of $0.06 \mathrm{wt} \%$ ) at different field strengths $E\left(\mathrm{~V}_{\mathrm{rms}} / \mu \mathrm{m}\right)$. The two-headed arrow indicates the field direction E. Gravity, $g$, is perpendicular to $E$. The Fourier transformations (FTs) of the corresponding images are shown as an inset (scale bar $=5 \mu \mathrm{m}$ ). The inset figures at $E=0.01$ and $0.018 \mathrm{~V}_{\mathrm{rms}} /$ $\mu \mathrm{m}$ are the magnified images of a string and $2 \mathrm{~d}$ array, respectively. The schematic image (right side) demonstrates the different steps of structure formation with increasing field strength from the top to bottom.

0.06 and $0.29 \mathrm{wt} \%$, respectively. At zero-field $(E=0)$, bacterial cells are diffusive and are distributed isotropically, which is evident from 2D CLSM images and their FT (the image at $E=$ 0 in Figures 1 and 2). At low field values, individual bacterium with its long axis orients along the field direction. The deviation from a zero-field isotropic ordering is clearly visible from the FT of its corresponding images (images at $E=0.0035$ and 0.009 in Figures 1 and 2, respectively). This oriented phase has 

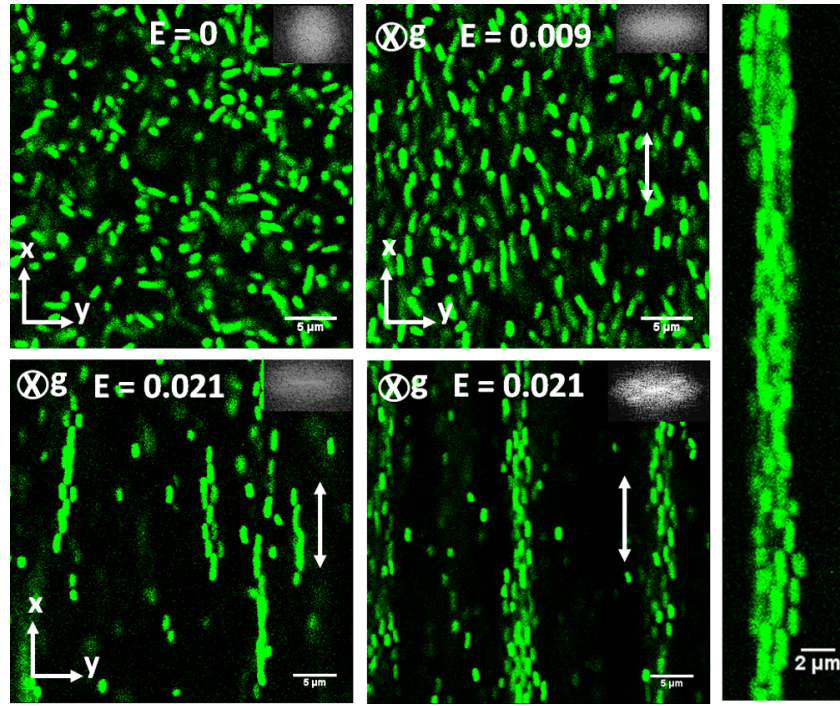

Figure 2. 2D CLSM images of bacterial suspension (at the concentration of $0.29 \mathrm{wt} \%$ ) at different field strengths. The FTs of the corresponding images are shown as an inset (scale bar $=5 \mu \mathrm{m}$ ). CLSM image (right side) corresponds to magnified version of a single column.

also been observed in other bacterial systems in a template assembly using DNA matrix ${ }^{3}$ or liquid crystal. ${ }^{2}$ This phase mimics paranematic ordering that has been observed in rodshaped synthetic colloids under an external electric field. ${ }^{16}$ At $E$ $=0.01 \mathrm{~V}_{\mathrm{rms}} / \mu \mathrm{m}$, the oriented bacteria form $1 \mathrm{D}$ chains along the field direction (Figure 1). These freely suspended chains break and again form spontaneously because of the competition between thermal fluctuation (on the order of $k_{\mathrm{B}} T$ ) and weak dipolar interaction. In chains, bacteria arrange in a zigzag manner at an average angle of $\theta=18^{\circ} \pm 1^{\circ}$ with the applied field direction to minimize the energy and maximize the attraction between dipoles (as shown in the inset image at $E=$ 0.01 in Figure 1). In the case of spherical particles under an external alternating field, the minimum energy (maximum attraction) occurs when the induced dipoles align along the field direction at an angle of $\theta=0 .{ }^{12,13}$ However, for anisotropic particles under an external electric field, the energy minimization for maximum dipolar attraction always occurs at $\theta>0$ as per previous experimental and theoretical studies. ${ }^{15-17}$ The value of $\theta$ varies depending upon the aspect ratio. These field-induced structures form instantaneously when the field is on and redisperse again to the homogeneous isotropic phase when the field is switched off. A demonstration of such reversibility is shown in Movie S1.

With further increase in electric field strength to $E=0.018$ $\mathrm{V}_{\text {rms }} / \mu \mathrm{m}$ (see Figure 1 ), more bacteria associate laterally with the $1 \mathrm{D}$ chains to form a $2 \mathrm{D}$ structure where each bacterium is surrounded by four nearest neighbors. Similar $2 \mathrm{D}$ arrays have also been observed at the higher bacterial concentration of 0.29 wt \% (see the first image at $E=0.021 \mathrm{~V}_{\mathrm{rms}} / \mu \mathrm{m}$ in Figure 2). In the later stage, we observe that as a function of time, these $2 \mathrm{D}$ structures grow and combine with other $2 \mathrm{D}$ structures to form large extended structures that mimic columns (see the second image at $E=0.021 \mathrm{~V}_{\mathrm{rms}} / \mu \mathrm{m}$ in Figure 2). The magnified image of a single column is shown in Figure 2. Even in some cases, where the samples are kept for a longer period under constant field values, the columns are found to span both sides of electrodes. The average width of these columns varies from 3 to
5 microns at this concentration. Bragg diffraction spots in the FT image of the columns (inset FT image at $E=0.021 \mathrm{~V}_{\mathrm{rms}} /$ $\mu \mathrm{m}$ ) indicate a crystalline arrangement of bacteria within a single column. This crystalline order is very clear when we look at the columnar structures at further high bacterial concentration.

In the next step, we obtain a 3D overview of these columnar structures by carrying out the field experiments at a comparable bacterial concentration as per Scheme 2. The field-induced

Scheme 2. Bacterial Suspension Is Kept between Two ITOCoated Cover Slips Separated by a Spacer of $120 \mu \mathrm{m}^{a}$

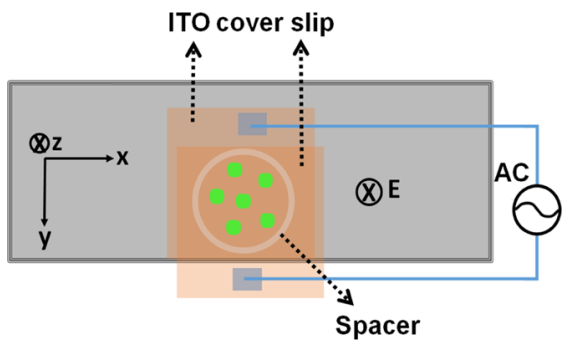

${ }^{a}$ We view the structure along the applied electric field direction (along $z$ ), which is perpendicular to the image $(x y)$ plane. Under an ac field, bacteria align along the field direction and look spherical in the image plane as shown in the schematic.

structural ordering of bacteria in the $x y$-image plane perpendicular to the field direction (along $z$ ) is shown in Figure 3A. At zero-field $(E=0)$, rod-shaped bacteria are diffusive and distributed in a homogeneous manner, which is consistent with the observations in Scheme 1. At a low field strength, the oriented bacteria and the bacterial chains look like spheres in the image plane (the second image at $E=0.01$ of Figure $3 \mathrm{~A}$ ). With further increase in field strength, we see the formation of clusters and the size of these clusters (i.e., the width of the columnar phase) increases with increase in the field strength from $E=0.015$ to $0.02 \mathrm{~V}_{\mathrm{rms}} / \mu \mathrm{m}$ (see Figure 3A). The $3 \mathrm{D}$ overview of these columnar structures is constructed from several $2 \mathrm{D}$ images along the $z$-axis at $E=0.02 \mathrm{~V}_{\mathrm{rms}} / \mu \mathrm{m}$, and the corresponding 3D image is shown in Figure 3B. Hence, we demonstrate that the columnar structures that we see in an $x y$-plane (in Figure 2) using Scheme 1 are $2 \mathrm{D}$ representations of $3 \mathrm{D}$ columnar structures that are elongated along the $z$-axis when we view along the field direction using Scheme 2 .

We further look at the field-induced assembly at a very high bacterial concentration of 2.9 wt \% (see Figure 4). At this high bacterial concentration, the zero-field structure is purely isotropic as is evident from the inset FT of the 2D CLSM image at $E=0$ (see Figure 4). With increasing field strength, we find transition from isotropic to columnar phase at $E=0.02$ $\mathrm{V}_{\text {rms }} / \mu \mathrm{m}$ via an oriented phase at $E=0.005 \mathrm{~V}_{\text {rms }} / \mu \mathrm{m}$ (see Figure 4). No intermediate chain phase is observed at this high bacterial concentration. The width of the columns varies between 15 and 20 microns. Moreover, in the columnar phase, we now clearly see the ordered arrangement of bacteria with centered rectangular unit cell, where a single bacterium is surrounded by four bacteria with average lattice constants $a=$ $1.1 \pm 0.3 \mu \mathrm{m}$ and $b=3.5 \pm 1 \mu \mathrm{m}$ (see the high and low magnification images at $E=0.02$ in Figure 4). The FT of this image exhibits distinct diffraction spots, which is evidence for the presence of long-range order. However, due to the high degree of polydispersity, Bragg spots are not very sharp and no 


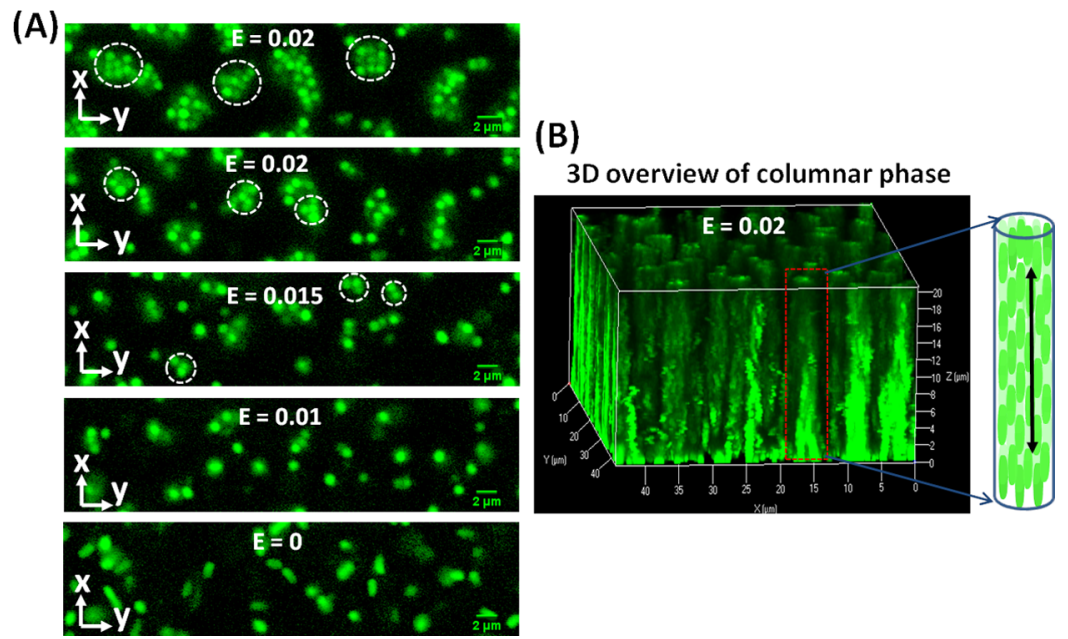

Figure 3. (A) 2D CLSM images at different field strengths at a bacterial concentration of $0.25 \mathrm{wt} \%$. The electric field is perpendicular to the image plane as per Scheme 2. (B) 3D image of the columnar phase (at $E=0.02 \mathrm{~V}_{\mathrm{rms}} / \mu \mathrm{m}$ ) constructed from several $2 \mathrm{D}$ images at an interval of 0.4 micron along the $z$-direction. The schematic in panel (B) represents that the oriented bacteria along the field direction are packed in a columnar structure. The two-headed arrow represents the field direction.
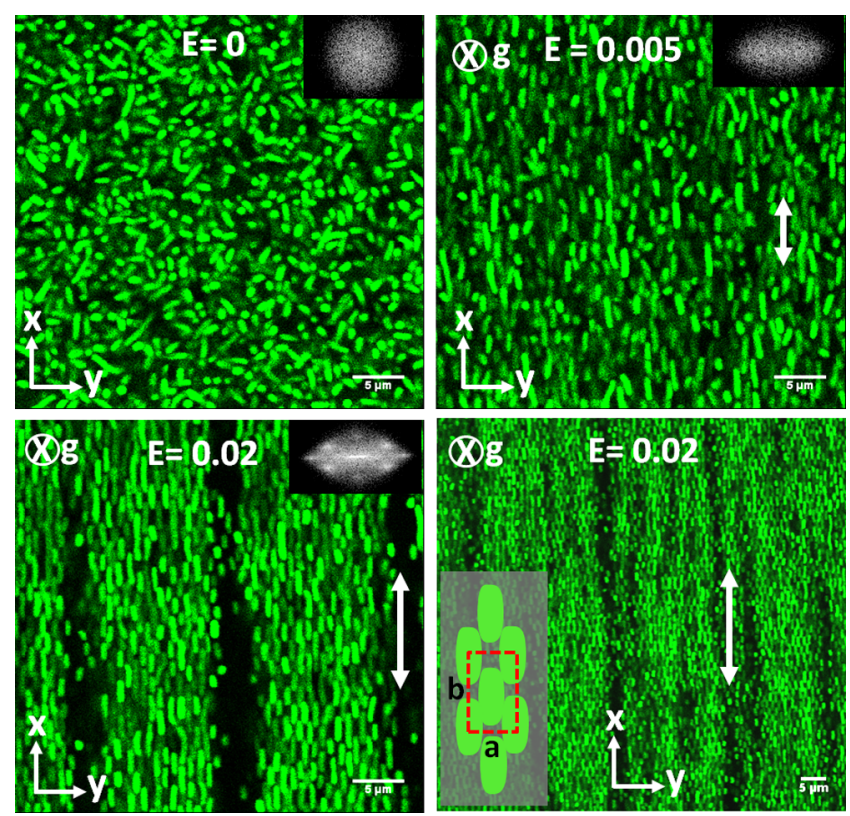

Figure 4. 2D CLSM images of the field-induced structure formation in bacterial suspension at a high concentration of $2.9 \mathrm{wt} \%$. At $E=0.02$ $\mathrm{V}_{\mathrm{rms}} / \mu \mathrm{m}$, two images correspond to high and low zooms, respectively. The two-headed arrow indicates the field direction $E$. Gravity, $g$, is perpendicular to $E$. The FTs of the corresponding images are shown as an inset. The scale bar indicates 5 micron.

higher-order reflections are observed. We could not pursue further quantitative characterizations on this crystalline phase.

We now summarize our experimental results through a qualitative phase diagram as a function of electric field strength and bacterial cell concentrations (see Figure 5). One can notice that at a given cell concentration, the structure formation under the ac field mostly depends on the strength of dipole moments, which can be tuned by changing the electric field strength. At a low concentration, the transition from the zero-field randomly oriented isotropic phase to the oriented phase is obtained above the field strength of $E>0.002 \mathrm{~V}_{\mathrm{rms}} / \mu \mathrm{m}$. When the field strength is further increased, $1 \mathrm{D}$ chains and $2 \mathrm{D}$ arrays are formed above $E>0.009$ and $0.016 \mathrm{~V}_{\mathrm{rms}} / \mu \mathrm{m}$, respectively. At a

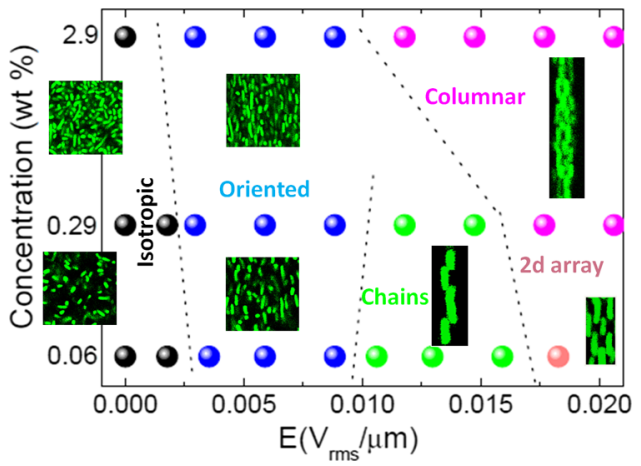

Figure 5. Phase diagram of different structures that form at different bacterial cell concentrations under an ac field at a frequency of $2 \mathrm{MHz}$.

higher bacterial concentration of 0.29 wt \%, the boundary of oriented phase slightly shifts to lower field values. This may be because the oriented phase is already favored at higher bacterial concentration. Thus, a lower field strength is required to orient the rod-shaped bacteria. It seems that the chain regime gets narrower with increasing bacterial concentration. For the highest bacterial concentration (at 2.9 wt \%), we find almost no intermediate chains and the oriented phase directly undergoes a transition to the columnar phase.

It must be noted that we currently know neither the details of effective interparticle interactions between bacteria in the presence of an external electric field nor the frequencydependent polarization mechanisms: both are necessary to know the details of mechanisms that govern the concentrationdependent electric-field-induced structure formation in our bacterial system. To understand the frequency-dependent polarization mechanisms, impedance spectroscopy will be carried out in future. We also plan to examine, in a future study, the effective interparticle interactions between bacteria under the presence of an external electric field.

In the present case, we can nevertheless discuss our experimental results based on the existing theoretical/ simulation studies on related topics. The oriented and the chain phases that we have observed in our electric field experiments have been explained in earlier theoretical/ 
simulation work on the behavior of biological cells under an external ac electric field. In these studies, the biological cells are modeled as particles covered with shells, and the interactions among the cells are treated in the dipole approximation. ${ }^{26,27}$ In the Brownian dynamics simulation work of Llamas et al., ${ }^{27}$ each cell (of spherical shape) is subjected to various forces such as electrical dipolar interaction, a short-range repulsive interaction, a viscous force, and a Brownian force. Through simulation, they observe two regimes: first, the cells form linear chains under an external electric field, and second, the chains condense into columnar structures. Although there is a qualitative agreement with respect to the formation of chains and columns, the exact structures of their chains and columns are different from ours because of different shapes. Moreover, these studies are limited to dilute concentration, and no such phase diagram between electric field strength and cell concentrations has been considered that can be used as a reference to understand our experimental results.

On the other hand, to a first approximation, if bacteria can be considered to be polarizable rods, then some of our results can be qualitatively compared with the past Monte Carlo simulations of monodisperse polarizable hard rod (of aspect ratio $=5$, which is similar to that in our case) based on a double-charge model. ${ }^{28}$ The simulation studies have predicted a phase diagram as a function of electric field strength and rod volume fraction, which contains a variety of stable phases such as nematic, strings (or chains), smectic $\mathrm{A}$, smectic $\mathrm{C}$, columnar, and other crystal phases AAA, HSC, X, and ABC. The observations of nematic and chains at low volume fraction and columns at high volume fraction in their simulation studies are qualitatively similar to our experimental findings where we see an oriented phase and chain phase at low bacterial concentration and a columnar phase at high bacterial concentration. However, in our experiments, we do not observe other higher-order stable phases such as smectic A, smectic B, and so forth as seen in the simulated phase diagram of hard rods. There may be different reasons for this disagreement. First, the interaction potential could be different. In the case of bacteria, the interaction potential is expected to be much softer than the polarizable hard rods. Second, the rod-shaped bacteria exhibit polydispersity in size. These may be the reasons why we do not observe higher-order crystalline phases in our bacterial systems. This is also true based on other earlier simulation studies $^{29}$ on polydisperse rod particles in the limit of infinite aspect ratio, where it has been shown that with a polydispersity of more than $18 \%$, only nematic and columnar phases are stable, whereas smectic phase is not.

Finally, it is important to discuss our observed results with respect to the similar studies previously reported on bacterial assembly. The oriented phase (similar to paranematic-like ordering), which we observe because of induced dipolar forces, had also been noticed in other living bacterial cells, such as Pseudomonas aeruginosa. In this work, the oriented phase was achieved through the elastic forces exerted by templated liquid crystalline (LC) matrix of concentrated DNA. ${ }^{3}$ In another study, it was also shown that Proteus mirabilis-flhDC bacterial cells associate to form $1 \mathrm{D}$ chains mediated by elastic forces from lyotropic nematic LC matrix. ${ }^{2}$ In a very recent work, nanowire arrays have been used as a matrix to obtain bacterial assembly (Sporomusa ovata cells) through an electrostatic interaction of bacteria with nanowires. ${ }^{5}$ However, in all of these studies, the concentration of bacterial cells is too low to observe any large aggregates of bacteria forming any ordered structures.
Moreover, these self-assembly methods do not have switchable functionality with an on/off action. It is also worth noting that the field-induced columnar structures, which we observe for the first time in our experiments, can mimic those detected in other biological systems such as rod viruses ${ }^{30}$ and $\mathrm{DNA}^{31}$ at larger concentrations. The advantages in our case are that these columnar phases can also be generated at low bacterial concentration using an external ac electric field and that the structures are fully reversible.

\section{CONCLUSIONS AND OUTLOOK}

We believe that our results unambiguously demonstrate how an external field can be used in a living bacterial system to generate different types of structures at varying length and time scales. Formation of quick and reversible structures in bacterial cells in the presence of an external ac electric field is very similar to electrorheological (ER) materials. ${ }^{32}$ Earlier electric field studies ${ }^{12-18}$ on different synthetic particles of different shapes have already shown the formations of $1 \mathrm{D}$ chains, $2 \mathrm{D}$ and $3 \mathrm{D}$ crystals and their potential applicability as model systems for ER fluids. The combined action of electrical and rheological properties of ER fluids has been proposed for applications in many devices such as clutches, brakes, valves, and photonics. On the other hand, some of the recent studies ${ }^{18}$ have demonstrated the design of novel hybrid biomaterials of $1 \mathrm{D}$ chain, 2D array composed of biological cells, and synthetic colloids, which will have potential applications in biosensors.

We thus believe that our studies on the field-induced structure formations in bacterial cells may help further to designing more complex hybrid biomaterials for specific biosensor applications. Because the structure formation does not require any genetic modification or re-engineering, our methods can also be applied to different shapes of bacteria and can also be combined with different types of functional colloids to design novel structures. Apart from this, our methods can also find interest in tissue engineering ${ }^{33}$ and construction of model biofilms ${ }^{34}$ where cells of different types can be assembled into $1 \mathrm{D}, 2 \mathrm{D}$, or $3 \mathrm{D}$ structures under an external ac electric field to construct artificial tissue.

\section{ASSOCIATED CONTENT}

\section{Supporting Information}

The Supporting Information is available free of charge on the ACS Publications website at DOI: 10.1021/acsomega.6b00541.

Details of bacterial culture and growth curve characterizations. Details of the determination of hydrodynamic radius using dynamic light scattering (PDF)

The demonstration of instantaneous and reversible field induced structure formation in deionized bacterial suspension at low concentration (AVI)

\section{AUTHOR INFORMATION}

\section{Corresponding Author}

*E-mail: pritimohanty02@gmail.com (P.S.M.).

ORCID $\odot$

Priti Sundar Mohanty: 0000-0001-8706-8005

\section{Present Address}

${ }^{\perp}$ Department of Physical Chemistry, Lund University, Sweden. Author Contributions

P.S.M. designed the study, analyzed the data, and wrote the paper. K.S., B.S., S. K. Barik, and K.J. characterized the materials 
and performed the experiments under the supervision of P.S.M. K.S. and P.B. contributed to data analysis and paper writing. S. K. Bhat, B.R.S., and M.S. contributed to the discussion and critically carried out the English correction.

\section{Author Contributions}

${ }^{\#}$ K.S. and P.B. contributed equally to this work.

\section{Funding}

P.S.M. and S. K. Bhat acknowledges SERB, Department of Science and Technology (India) (ref: SB/S3/CE/042/2015), India, for partial support, and P.S.M. and K.S. acknowledges Biotechnology Ignition Grant (BIG), Department of Biotechnology (India) (ref: BIRAC/KIIT0055/BIG-06/14) for partial funding.

\section{Notes}

The authors declare no competing financial interest.

\section{ACKNOWLEDGMENTS}

P.S.M. acknowledges Dr. B. Ravindran (Emeritus Scientist, Institute of Life Science, Bhubaneswar) for providing access to the confocal microscope facility. P.S.M. acknowledges Prof. Anand Yethiraj for useful suggestions and critical reading of the revised manuscript.

\section{REFERENCES}

(1) Zhou, S.; Sokolov, A.; Lavrentovich, O. D.; Aranson, I. S. Proc. Natl. Acad. Sci. U.S.A. 2014, 111, 1265.

(2) Mushenheim, P. C.; Trivedi, R. R.; Tuson, H. H.; Weibel, D. B.; Abbott, N. L. Soft Matter 2014, 10, 88.

(3) Smalyukh, I. I.; Butler, J.; Shrout, J. D.; Parsek, M. R.; Wong, G. C. L. Phys. Rev. E: Stat., Nonlinear, Soft Matter Phys. 2008, 78, 030701.

(4) Gloag, E. S.; Turnbull, L.; Huang, A.; Vallotton, P.; Wang, H.; Nolan, L. M.; Mililli, L.; Hunt, C.; Lu, J.; Osvath, S. R.; Monahan, L. G.; Cavaliere, R.; Charles, I. G.; Wand, M. P.; Gee, M. L.; Prabhakar, R.; Whitchurch, C. B. Proc. Natl. Acad. Sci. U.S.A. 2013, 110, 11541.

(5) Sakimoto, K. K.; Liu, C.; Lim, J.; Yang, P. Nano Lett. 2014, 14, 5471.

(6) Copeland, M. F.; Weibel, D. B. Soft Matter 2009, 5, 1174.

(7) Lu, C.; Möhwald, H.; Fery, A. Soft Matter 2007, 3, 1530.

(8) van Blaaderen, A.; Ruel, R; Wiltzius, P. Nature 1997, 385, 321.

(9) Yan, Q.; Chen, A.; Chua, S. J.; Zhao, X. S. J. Nanosci. Nanotechnol. 2006, 6, 1815.

(10) Faraudo, J.; Andreu, J. S.; Calero, C.; Camacho, J. Adv. Funct. Mater. 2016, 26, 3837.

(11) Snezhko, A.; Aranson, I. S. Nat. Mater. 2011, 10, 698.

(12) Yethiraj, A.; van Blaaderen, A. Nature 2003, 421, 513.

(13) Mohanty, P. S.; Bagheri, P.; Nöjd, S.; Yethiraj, A.; Schurtenberger, P. Phys. Rev. X 2015, 5, 011030.

(14) Chaudhary, K.; Juárez, J. J.; Chen, Q.; Granick, S.; Lewis, J. A. Soft Matter 2014, 10, 1320.

(15) Singh, J. P.; Lele, P. P.; Nettesheim, F.; Wagner, N. J.; Furst, E. M. Phys. Rev. E: Stat., Nonlinear, Soft Matter Phys. 2009, 79, 050401R.

(16) Kuijk, A.; Troppenz, T.; Filion, L.; Imhof, A.; van Roij, R.; Dijkstra, M.; van Blaaderen, A. Soft Matter 2014, 10, 6249.

(17) Crassous, J. J.; Mihut, A. M.; Wernersson, E.; Pfleiderer, P.; Vermant, J.; Linse, P.; Schurtenberger, P. Nat. Commun. 2014, 5, 5516.

(18) Gupta, S.; Alargova, R. G.; Kilpatrick, P. K.; Velev, O. D. Langmuir 2010, 26, 3441.

(19) Kang, K.; Dhont, J. K. G. Europhys. Lett. 2008, 84, 14005.

(20) Kang, K.; Dhont, J. K. G. Soft Matter 2010, 6, 273.

(21) Siebman, C.; Velev, O. D.; Slaveykova, V. I. Biosensors 2015, 5, 319.

(22) Saito, M.; Schwan, H. P.; Schwarz, G. Biophys. J. 1966, 6, 313.

(23) Flemming, H.-C.; Wingender, J. Nat. Rev. Microbiol. 2010, 8, 623.

(24) Steenackers, H.; Hermans, K.; van der Leyden, J.; De Keersmaecker, S. C. J. Food Res. Int. 2012, 45, 502.
(25) Das, J. K.; Mishra, D.; Ray, P.; Tripathy, P.; Beuria, T. K.; Singh, N.; Suar, M. Gut Pathog. 2013, 5, 11.

(26) Schwarz, G.; Saito, M.; Schwan, H. P. J. Chem. Phys. 1965, 43, 3562.

(27) Llamas, M.; Giner, V.; Sancho, M. J. Phys. D: Appl. Phys. 1998, $31,3160$.

(28) Troppenz, T.; Filion, L.; van Roij, R.; Dijkstra, M. J. Chem. Phys. 2014, 141, 154903.

(29) Bates, M. A.; Frenkel, D. J. Chem. Phys. 1998, 109, 6193.

(30) Bawden, F. C.; Pirie, N. W.; Bernal, J. D.; Fankuchen, I. Nature 1936, 138, 1051.

(31) Livolant, F.; Leforestier, A. Prog. Polym. Sci. 1996, 21, 1115.

(32) Wen, W.; Huang, X.; Sheng, P. Soft Matter 2008, 4, 200.

(33) Markx, G. H. Organogenesis 2008, 4, 11.

(34) Abidin, Z. Z.; Downes, L.; Markx, G. H. Biotechnol. Bioeng. 2007, 96, 1222. 